\title{
DILEPTONS AND OPEN CHARM: PROBES OF CHIRAL RESTORATION
}

\author{
K. GALLMEISTER ${ }^{1}$, B. KÄMPFER ${ }^{2}$ AND S. ZSCHOCKE ${ }^{2,3}$ \\ 1 Universität Giessen, D-35392 Giessen, Germany \\ ${ }^{2}$ Forschungszentrum Rossendorf/Dresden, PF 510119, D-01314 Dresden, Germany \\ 3 Technische Universität Dresden, D-01068 Dresden, Germany
}

We summarize the status of electromagnetic probes of strongly interacting matter produced in relativistic heavy-ion collisions at CERN-SPS with respect to indications of chiral symmetry restoration. Explorative results for studying the open charm dynamics at BNL-RHIC are presented.

\section{Introduction}

The dilepton emission rate of thermalized matter reads ${ }^{1,2}$

$$
\frac{d R}{d^{4} Q}=\frac{\alpha^{2}}{3 \pi^{3} Q^{4}} \operatorname{Im} \Pi_{\mu \nu}^{R} L^{\mu \nu} f_{B}(Q \cdot u / T),
$$

where $Q$ denotes the 4-vector of the pair, $L^{\mu \nu}=Q^{\mu} Q^{\nu}-Q^{2} g^{\mu \nu}$, and $f_{B}$ stands for the thermal (Bose) distribution; $u$ is the 4velocity of the medium. The central quantity, $\Pi_{\mu \nu}^{R}$, is the retarded photon self-energy in the medium which is related to the currentcurrent correlator via

$$
\Pi_{\mu \nu}^{R}=i \int d^{4} x \mathrm{e}^{i Q x} \Theta\left(x_{0}\right)\left\langle\left\langle\left[J_{\mu}(x), J_{\nu}(0)\right]\right\rangle\right\rangle,
$$

where $\langle\langle\cdots\rangle\rangle$ means the thermal average with respect to the medium with temperature $T$ and baryon density $n ; J_{\mu}$ is the electromagnetic current operator. Also the real photon emission rate is related to $\operatorname{Im} \Pi_{\mu \nu}^{R} 2,3$. Therefore, the electromagnetic probes are sensitive to the medium properties. Since the absorption probability of such probes, once emitted, is small they carry information on the full evolution, in particular also on the early hot, dense stages in heavy-ion collisions.

\section{Electromagnetic signals at CERN-SPS}

The dilepton emission rate, in dilute gas approximation, contains the model-

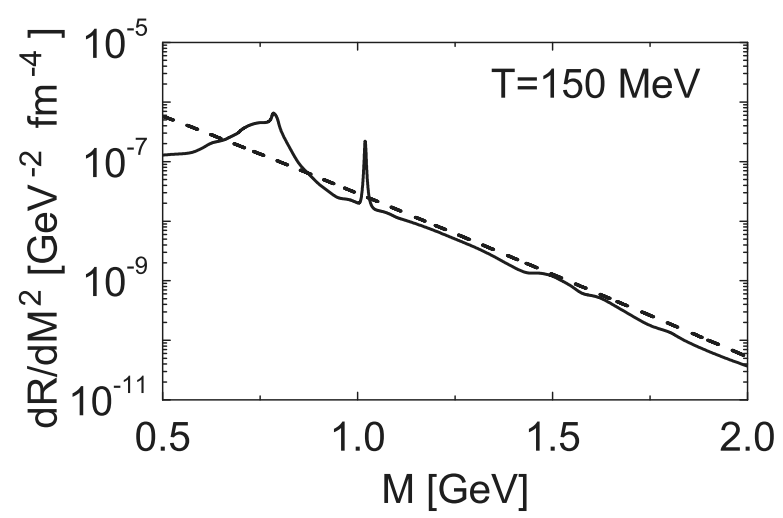

Figure 1. Dilepton emission rates of strongly interacting matter at temperature $T=150 \mathrm{MeV}$. The solid curve is based on Eq. (3), while the dashed line is the $\bar{q} q$ annihilation rate.

independent leading terms

$$
\frac{d R}{d^{4} Q}=\frac{4 \alpha^{2} f_{B}}{(2 \pi)^{2}}\left\{\rho^{e . m .}-\epsilon\left(\rho^{V}-\rho^{A}\right)+n \mathcal{T}\right\},
$$

where $\mathcal{T}$ is related to the nucleon matrix element of the current-current commutator, $\epsilon=T^{2} /\left(6 f_{\pi}^{2}\right)$ in the chiral limit, and

$$
\begin{aligned}
\rho^{e . m .}= & \frac{1}{12 \pi^{2}} \sigma_{e^{+} e^{-} \rightarrow \text { hadrons }} / \sigma_{e^{+} e^{-} \rightarrow \mu^{+} \mu^{-}}, \\
\rho^{V}= & \frac{1}{12 \pi^{2}}\left[\sum_{i} \sigma_{e^{+} e^{-} \rightarrow 2 i \pi}\right] / \sigma_{e^{+} e^{-} \rightarrow \mu^{+} \mu^{-}}, \\
\rho^{A}= & \frac{8 \pi m_{\tau}^{3}}{G_{F}^{2} \cos ^{2} \Theta_{C}\left(m_{\tau}^{2}+2 Q^{2}\right)\left(m_{\tau}^{2}-Q^{2}\right)} \\
& \sum_{i} d \Gamma_{\tau \rightarrow \nu_{\tau}(2 i+1) \pi} / d Q^{2}
\end{aligned}
$$

(cf. Ref. ${ }^{4}$ for details). This rate is displayed 

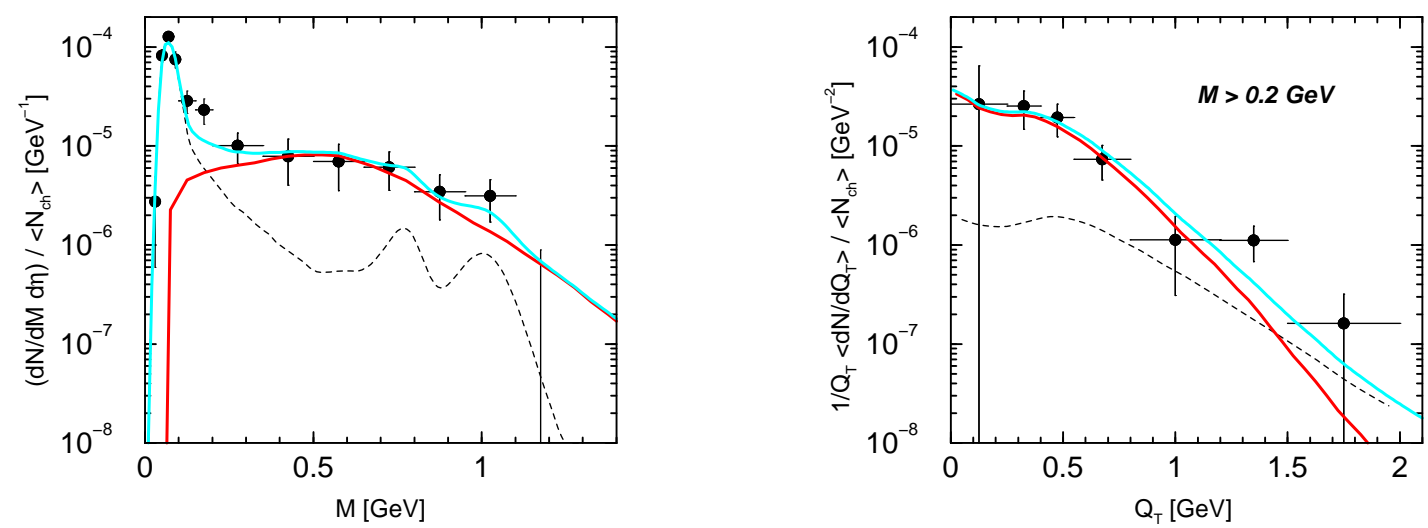

Figure 2. Dielectron spectra for the reaction $\mathrm{Pb}(40 \mathrm{AGeV})+\mathrm{Au}$ (left panel: invariant mass spectrum, right panel: transverse momentum spectrum, dashed curves: hadronic cocktail, red curves: thermal yield, cyan (upper) curves: sum of these contributions). Data from Ref. ${ }^{8} ;\langle T\rangle=145 \mathrm{MeV}$.
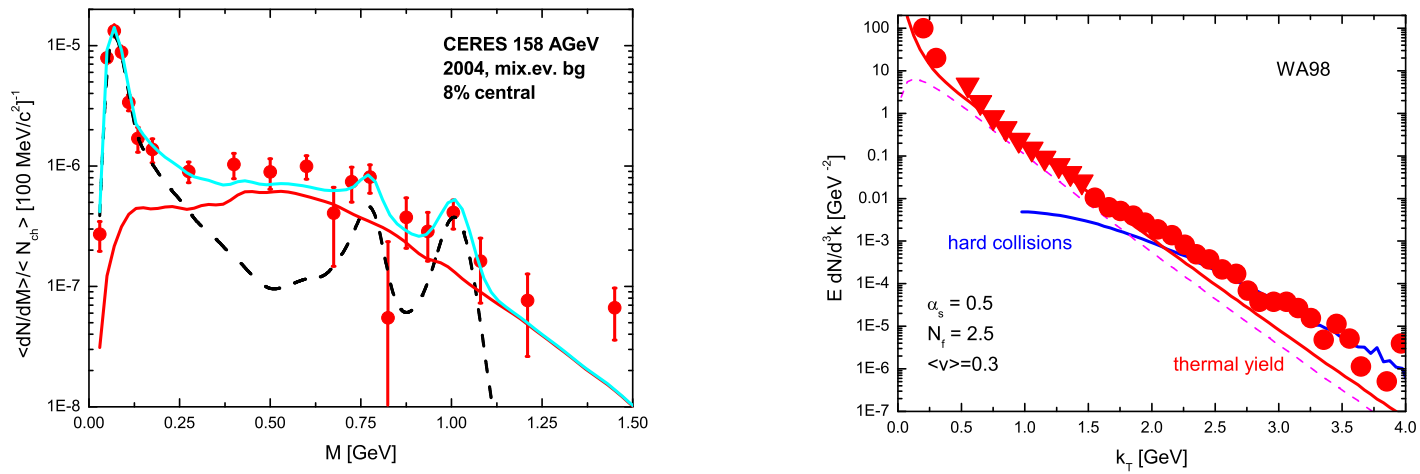

Figure 3. Left panel: Dielectron spectrum for the reaction $\mathrm{Pb}(158 \mathrm{AGeV})+\mathrm{Au}$ (line codes as in Fig. 2). Data from Ref. ${ }^{9} ;\langle T\rangle=170 \mathrm{MeV}$. Right panel: Photon spectrum for the reaction $\mathrm{Pb}(158 \mathrm{AGeV})+\mathrm{Pb}$ (blue curve: perturbative hard QCD contribution, dashed curve: lowest-order thermal yield, red solid curve: thermal yield corrected by resumed higher-order ${ }^{7}$ ). Data from Ref. ${ }^{10}$ (error bars are omitted, triangles depict upper limits); $\langle T\rangle=170 \mathrm{MeV}$; averaged transverse expansion velocity $\langle v\rangle=0.3 c$.

in Fig. 1 for $n=0$. It coincides remarkably well with the Born $\bar{q} q$ annihilation rate for invariant masses $M>1 \mathrm{GeV}$ in a wide range of $T$; the Born rate in turn is in good agreement with the lattice QCD evaluation ${ }^{5}$. A radical point of view is to use the $\bar{q} q$ rate as convenient parametrization in the full range of invariant masses and temperatures with the arguing that chiral symmetry arguments ${ }^{1}$ support a reshuffling of strength in the $\rho-\omega$ region such that also there a featureless continuum describes the emissivity of strongly interacting matter. Moreover, in Ref. ${ }^{6}$ it has been shown that instead of the use of the de- tailed space-time evolution the replacement $\int d^{4} x d R(T, n) / d^{4} Q \rightarrow \mathcal{N} d R(\langle T\rangle) / d^{4} Q$ delivers a consistent description of the dilepton experiments CERES, HELIOS-3, NA38, NA50 at CERN-SPS with suitably adjusted normalization $\mathcal{N}$ and space-time averaged temperature $\langle T\rangle$. In Figs. 2 and 3 we show two examples for very recent data in the lowmass region. In the intermediate-mass region, charm contributions (see below) become important and the high-mass region is dominated by the Drell-Yan yield. In particular the forthcoming NA60 data are expected to shed further light on the role of the charm 
contribution.

When supplementing, in the same sprit, the Born rate for photons by the collinear enhancement factor ${ }^{7}$ one gets equally well the description of the recent WA98 data ${ }^{10}$, see Fig. 3.

Advanced models (cf. Refs. ${ }^{1,2,3}$ ) resolve the details of the space-time evolution and make contact to the equation of state ${ }^{12}$ but do not improve the agreement with data. A particularly important result is that the needed maximum (i.e., initial) temperature is above the deconfinement temperature.

\section{Charm at RHIC}

To describe the intermediate-mass dilepton spectrum at CERN-SPS one must take into account the contribution of correlated semileptonic decays of open charm mesons ${ }^{6}$. At RHIC energies this contribution is expected to dominate by far. Therefore, the correct treatment of charm is important. Experimental data ${ }^{11}$ on the inclusive single electron spectra in $p p$ collisions may serve for adjustment purposes. In Fig. 4 the corresponding spectra are displayed. The charm and bottom cross sections are 650 and $4.3 \mu \mathrm{b}$, respectively. Here we have used a common $K$ factor both for open charm and bottom of the constant value of 5 .

As in the first reference of Ref. ${ }^{6}$ the gluon-radiative energy loss of charmed quarks is parameterized in a geometrical model and the decay single-electron spectra are compared with data in Fig. 5 for heavy-ion collisions $\mathrm{Au}+\mathrm{Au}$ at $\sqrt{s_{N N}}=200 \mathrm{GeV}$. Similar to the results at $130 \mathrm{GeV}^{6}$, the energy loss becomes noticeable and thus measurable at large momenta, where, however, precision data are still lacking. While the effect of the energy loss for the initial conditions with undersaturated quark-gluon medium is hardly visible, the changes due to energy loss in a saturated quark-gluon fluid are somewhat larger, but still not very strong. We would

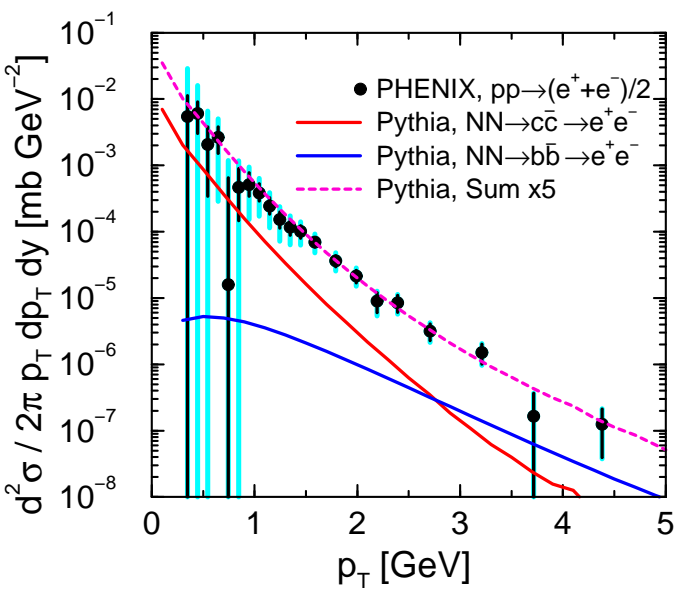

Figure 4. Single electron spectrum (data [nonphotonic sources] from Ref. ${ }^{11}$ ) for the reaction $\mathrm{p}+\mathrm{p}$ at $\sqrt{s}=200 \mathrm{GeV}$ compared with results of PYTHIA $(\mathrm{PDF}=\mathrm{CTEQ} 5 \mathrm{~L})$ with default parameters unless hybrid fragmentation and $\left\langle k_{\perp}^{2}\right\rangle=2.5 \mathrm{GeV}^{2}$, which describe charged hadron spectra fairly well ${ }^{13}$. The transverse momentum spectra ${ }^{14}$ of $D^{0}$ and $\bar{D}^{0}$ mesons in the reaction $\mathrm{d}+\mathrm{Au}$ at $\sqrt{s_{N N}}=200 \mathrm{GeV}$, however, are underestimated in normalization and slope for $p_{\perp}>6 \mathrm{GeV} / \mathrm{c}^{15}$ for these settings.

like to add that these energy loss scenarios are probably an upper limit, since we did not include the dead cone effect ${ }^{16}$ and neglected $\log$ terms. The dielectron spectrum is more sensitive to the energy loss of charm quarks, see Fig. 5. The forthcoming dilepton data, therefore, may be essential to pin down quantitatively the energy loss of charm quarks.

\section{Summary}

At CERN-SPS the thermal electromagnetic radiation (dileptons and real photons) off the fireball of strongly interacting matter has been identified in heavy-ion collisions. It is compatible with the assumption of achieving maximum temperatures of $\mathcal{O}(200) \mathrm{MeV}$, i.e. above the deconfinement temperature. The low-mass dilepton spectrum needs a drastic reshaping in the resonance region, compatible with expectations from chiral restoration. The first electromagnetic signals in heavyion experiments at RHIC are compelling but 

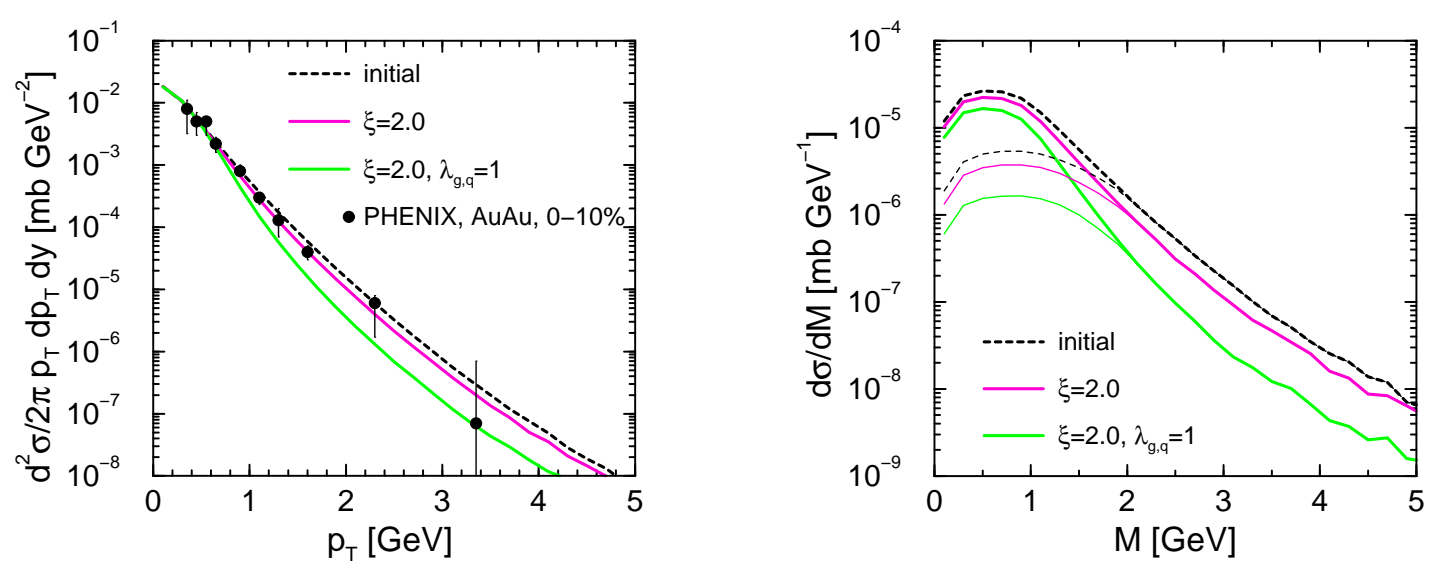

Figure 5. Left panel: Single electron spectra from semileptonic open charm decays for the reaction $\mathrm{Au}+\mathrm{Au}$ at $\sqrt{s_{N N}}=200 \mathrm{GeV}$ for various assumptions on the energy loss parameterized by the strenght factor $\xi$ as in Ref. $^{6}$ (dashed curve: no energy loss; magenta curve: maximum temperature $550 \mathrm{MeV}$ and initial quark-gluon undersaturation; green curve: the same maximum temperature but initial saturation). Data (non-photonic sources) from Ref. ${ }^{11}$. Right panel: Corresponding dilepton spectra within the PHENIX acceptance with minimum single electron momentum of $500 \mathrm{MeV} / \mathrm{c}$ (solid curves) or $1 \mathrm{GeV} / \mathrm{c}$ (thin curves).

need higher precision to arrive at firm conclusions and to compare with the many existing predictions. The important role of heavy flavor dynamics seems to be confirmed. Besides dileptons and photons, diphotons should be mentioned as interesting probe ${ }^{17}$.

\section{Acknowledgments}

This work is supported in part by BMBF 06DR121 and GSI.

\section{References}

1. R. Rapp and J. Wambach, Adv. Nucl. Phys. 25, 1 (2000).

2. C. Gale and K.L. Haglin, hep-ph/ 0306098.

3. T. Peitzmann and M. Thoma, Phys. Rep. 364, 175 (2002).

4. Z. Huang, Phys. Lett. B 361, 131 (1995).

5. F. Karsch et al., Phys. Lett. B 530, 147 (2002).

6. K. Gallmeister et al., Nucl. Phys. A 715, 705 (2003); Nucl. Phys. A 698, 424 (2002); Nucl. Phys. A 688, 939
(2001); Phys. Rev. C 62, 057901 (2000); Phys. Lett. B 473, 20 (2000).

7. P. Arnold et al., JHEP 0112, 009 (2001).

8. D. Adamova et al. (CERES), Phys. Rev. Lett. 91, 042301 (2003).

9. A. Marin et al. (CERES), J. Phys. G 30, S209 (2004).

10. M.M. Aggarwal et al. (WA98), Phys. Rev. Lett. 93, 022301 (2004).

11. S. Kelly et al. (PHENIX), nucl-ex/ 0403057, J. Phys. G 30, S1189 (2004).

12. A. Peshier et al., Phys. Rev. D 66, 094003 (2002); Phys. Rev. C 61, 045203 (2000); Phys. Rev. D 54, 2399 (1996).

13. W. Cassing, K. Gallmeister, C. Greiner, Nucl. Phys. A 735, 277 (2004).

14. J. Adams et al. (STAR), nucl-ex/ 0407006.

15. M.X. Liu, nucl-ex/0405034.

16. R. Thomas et al., hep-ph/0405189.

17. J. Casalderry-Solana and E.V. Shuryak, hep-ph/0408178;

M. Hentschel et al., Z. Phys. C 75, 333 (1997). 